\title{
DEFINIÇÃO DA DIMENSÃO DO INDIVÍDUO SOLO E DETERMINAÇÃO DO NÚMERO DE AMOSTRAS SIMPLES NECESSÁRIO À SUA REPRESENTAÇÃO(1)
}

\author{
André Guarçoni M. ${ }^{(2)}$, Víctor Hugo Alvarez V. ${ }^{(3)}$, Roberto Ferreira \\ Novais $^{(3)}$, Reinaldo Bertola Cantarutti ${ }^{(3)}$, Hélio Garcia Leite ${ }^{(4)}$ \\ \& Francisco Morel Freire ${ }^{(5)}$
}

\begin{abstract}
RESUMO
Uma população é composta por indivíduos, e a amostragem correta dos indivíduos estima adequadamente as características da população. Porém, para avaliar a fertilidade do solo, quem seria o indivíduo solo (unidades de amostra) e qual sua dimensão? Com o objetivo de definir a dimensão do indivíduo solo componente de determinada população, sob plantio direto (PD) ou sob plantio convencional antes (PCAA) ou depois da aração (PCDA), visando avaliar a fertilidade do solo e desenvolver um método de amostragem de solos, determinando o número de amostras simples necessário à formação de uma amostra composta que caracterize o indivíduo solo (unidade de amostra), foram coletadas amostras simples de solo $(5,4 \mathrm{~cm}$ de diâmetro $\times 10 \mathrm{~cm}$ de profundidade) sobre as semidiagonais de cinco hexágonos delimitados sobre cada uma das áreas selecionadas para amostragem (PD, PCAA e PCDA). Os hexágonos de amostragem apresentavam $2 \mathrm{~m}$ de lado e, em cada uma das seis semidiagonais dos mesmos, foram coletadas dez amostras simples de solo ( 55 por hexágono) nas seguintes distâncias, a partir de uma amostra simples central, medidas até o centro do orifício de coleta: 12,$5 ; 25,0 ; 37,5 ; 50,0 ; 75,0 ; 100,0 ; 112,5 ; 150,0$ e $200,0 \mathrm{~cm}$, sendo a amostra simples central comum às seis semidiagonais. Foram determinados o $\mathrm{pH}\left(\mathrm{H}_{2} \mathrm{O}\right)$, os teores de $\mathrm{P}$ e K disponíveis e de $\mathrm{Ca}^{2+}$ e $\mathrm{Mg}^{2+}, \mathrm{H}+\mathrm{Al}$, P-rem e a matéria orgânica. As amostras simples foram agrupadas sucessivamente a partir do centro do hexágono, formando nove unidades de amostra: A (até 18,75), B (até 31,25), C (até 43,75), D (até 56,25), E (até 81,25), F (até 106,25), G (até 118,75), H (até 156,25) e I (até $206,25 \mathrm{~cm}$ ). Foi realizada análise de regressão das médias e dos desvios-padrão das
\end{abstract}

\footnotetext{
(1) Parte da Tese de Doutorado do primeiro autor. Recebido para publicação em julho de 2005 e aprovado em outubro de 2006.

(2) Pesquisador do Instituto Capixaba de Pesquisa. Assistência Técnica e Extensão Rural - INCAPER. CRDR-ES BR 262 Km 94 CEP 29375-000 Venda Nova do Imigrande (ES). E-mail: guarconi@incaper.es.gov.br

(3) Professor do Departamento de Solos, Universidade Federal de Viçosa - UFV. CEP 36571-000 Viçosa (MG). E-mails: vhav@ufv.br; rfnovais@ufv.br; cantarutti@ufv.br

(4) Professor do Departamento de Engenharia Florestal, UFV. E-mail: hgleite@ufv.br

(5) Pesquisador da EPAMIG-MG. Centro Tecnólogico do Centro-Oeste - CTCO. Rod. MG 424, Km 64, CEP 35701-970 Sete Lagoas (MG). E-mail: francisco.morel@epamig.br
} 
características avaliadas, considerando possíveis dimensões da unidade de amostra de solo. A partir dos resultados obtidos, pôde-se concluir que: para a caracterização do indivíduo solo (unidade de amostra), devem-se coletar 25 amostras simples, necessárias à formação de uma amostra composta representativa, num hexágono de $68,75 \mathrm{~cm}$ de lado e área de $1,228 \mathrm{~m}^{2}$.

Termos de indexação: amostragem, unidade de amostra, variabilidade superficial.

\title{
SUMMARY: DETERMINATION OF A SOIL INDIVIDUAL AND THE NUMBER OF SINGLE SAMPLES REQUIRED TO REPRESENT IT
}

\begin{abstract}
A population consists of individuals and a correct sampling of the individuals estimates population characteristics adequately. However, to evaluate soil fertility, what would be the soil individual (sample units), and of what size? The objective of this study was to determine the dimension of the individual soil component of a particular population, in three sampling areas: under no-till or under conventional tillage before or after plowing, and to evaluate soil fertility and develop a soil sampling method, determining the number of single samples required to form a composite sample that would characterize a soil individual (sample unit). Single soil samples $(5.4 \mathrm{~cm}$ diameter $x 10 \mathrm{~cm}$ deep) were collected along the semi-diagonals of five hexagons outlined on the selected sampling areas. The sampling hexagons had $2 \mathrm{~m}$ long sides and from each of the six semi-diagonals, 10 single soil samples (55 per hexagon) were collected at the distances $12.5 ; 25.0 ; 37.5 ; 50.0 ; 75.0$; 100.0; 112.5; 150.0 and $200.0 \mathrm{~cm}$, measured from a simple central sample, to the center of the sampling spot. The single central sample was the same for the six semi-diagonals. Soil $\mathrm{pH}\left(\mathrm{H}_{2} \mathrm{O}\right)$, available $\mathrm{P}$ and $\mathrm{K}, \mathrm{Ca}^{2+}$ and $\mathrm{Mg}^{2+}$ concentrations and $\mathrm{H}+\mathrm{Al}$, equilibrium- $\mathrm{P}$ and organic matter were determined. The single samples were grouped successively from the hexagon center and formed nine sampling units: A (up to 18.75), B (up to 31.25), $C$ (up to 43.75), D (up to 56.25), E (up to 81.25), F (up to 106.25), G (up to 118.75), H (up to 156.25) and I (up to $206.25 \mathrm{~cm}$ ). Regression analysis of the means and standard deviations of the evaluated characteristics was performed considering the possible dimensions of the soil sample unit. Based on the results, the conclusion was drawn that 25 simple samples must be collected to characterize a soil individual (sample unit), for the formation of a representative composite sample in a $68.75 \mathrm{~cm}$-sided hexagon with an area of $1.228 \mathrm{~m}^{2}$.
\end{abstract}

Index terms: sampling, sample unit, spatial variability.

\section{INTRODUÇÃO}

Uma população é um conjunto de indivíduos com pelo menos uma característica comum, sobre a qual se deseja obter informações. Uma amostra da população é um subconjunto ou parte da população, necessariamente finito, formado por indivíduos (unidades de amostra). A análise estatística da amostra é feita com base nos dados das unidades de amostra ou indivíduos (Cochran, 1965).

$\mathrm{Na}$ avaliação da fertilidade média do solo (agricultura tradicional), a amostra comumente utilizada para representar a área a ser corrigida e, ou, adubada é a "amostra composta". Cada amostra composta é formada pela mistura homogênea de um número predefinido de "amostras simples" de pequeno volume. Na agricultura de precisão, são coletadas, também, "amostras simples" de pequeno volume, na maioria das vezes, em um retículo limitado ao campo de produção, as quais são analisadas individualmente.
Dessa forma, apenas pela facilidade de coleta, as amostras simples estariam sendo utilizadas como unidades de amostra (indivíduos solo) componentes da população, assumindo-se, com grande otimismo, que os pequenos volumes de solo coletados nos dois casos representem corretamente o seu entorno, mas sem nenhuma evidência científica para isso. Assim, para avaliar a fertilidade do solo, tanto na agricultura tradicional quanto na agricultura de precisão, o "indivíduo solo" (unidade de amostra) pode ser definido como a menor área (considerando definida profundidade) na qual se deve amostrar o solo para caracterizar a fertilidade efetivamente explorada por uma planta ou por um grupo de plantas (fertilidade local média), sendo suas dimensões laterais grandes o bastante para incluir variações representativas a curtas distâncias na composição do solo (meso e microvariações).

Neste caso, porém, quem seriam os verdadeiros indivíduos e qual sua dimensão? Além disso, a coleta 
do indivíduo solo (unidades de amostra) poderia gerar benefícios para a amostragem, tanto na agricultura tradicional quanto na agricultura de precisão?

Este trabalho teve como objetivos: definir a dimensão do indivíduo solo, componente de determinada população, sob plantio direto ou sob plantio convencional antes ou depois da aração, visando avaliar a fertilidade do solo e desenvolver um método de amostragem de solos, determinando o número de amostras simples necessário à formação de uma amostra composta que caracterize o indivíduo solo (unidade de amostra).

\section{MATERIAL E MÉTODOS}

Foram coletadas amostras simples, com trado de $5,4 \mathrm{~cm}$ de diâmetro, na profundidade de 0 a $10 \mathrm{~cm}$, em um Cambissolo Háplico Argiloso (14, 8, 22, 56 \% de areia grossa, areia fina, silte e argila, respectivamente). A área experimental estava situada na UFV-Agronomia. As amostras simples foram coletadas em dois tipos de manejo: plantio direto (PD) e plantio convencional (aração - gradagem). No plantio convencional, foram utilizados dois momentos de coleta: antes da aração (PCAA) e depois da aração (PCDA). Desta forma, foram individualizadas três situações de manejo-coleta (PD, PCAA e PCDA).

No PD, sucessão milho - feijão, que apresentava sete anos desde a instalação, as amostras simples de solo foram coletadas no momento em que estava sendo cultivado milho no espaçamento de 0,90 m entrelinhas. Para o PCAA, as amostras simples de solo foram coletadas logo após a colheita de milho, cultivado no espaçamento de 0,90 m entrelinhas, em uma área onde se cultivava milho havia alguns anos e estava localizada a, aproximadamente, $50 \mathrm{~m}$ da área do PD. O PCDA estava localizado em uma área na qual vinham sendo cultivados trigo e soja por quatro anos em sucessão, localizada a, aproximadamente, $100 \mathrm{~m}$ da área do PCAA.

A coleta das amostras simples de solo foi realizada nas diagonais de um hexágono de $2 \mathrm{~m}$ de lado. Em cada semidiagonal do hexágono, foram coletadas dez amostras simples, com a amostra simples central comum às seis semidiagonais, gerando um total de 55 amostras simples para cada hexágono de amostragem. As distâncias foram medidas a partir do centro de cada orifício de coleta (Figura 1).

Foram demarcados cinco hexágonos em cada unidade de amostragem, correspondentes a cada situação de manejo-coleta: PD (275 amostras simples), PCAA (275 amostras simples) e PCDA (275 amostras simples). Em todos os casos, o ponto central de cada hexágono de amostragem foi escolhido de forma aleatória dentro das unidades de amostragem.

Considerando o valor da menor distância de coleta $(12,5 \mathrm{~cm})$ (Figura 1), uma vez que as outras distâncias

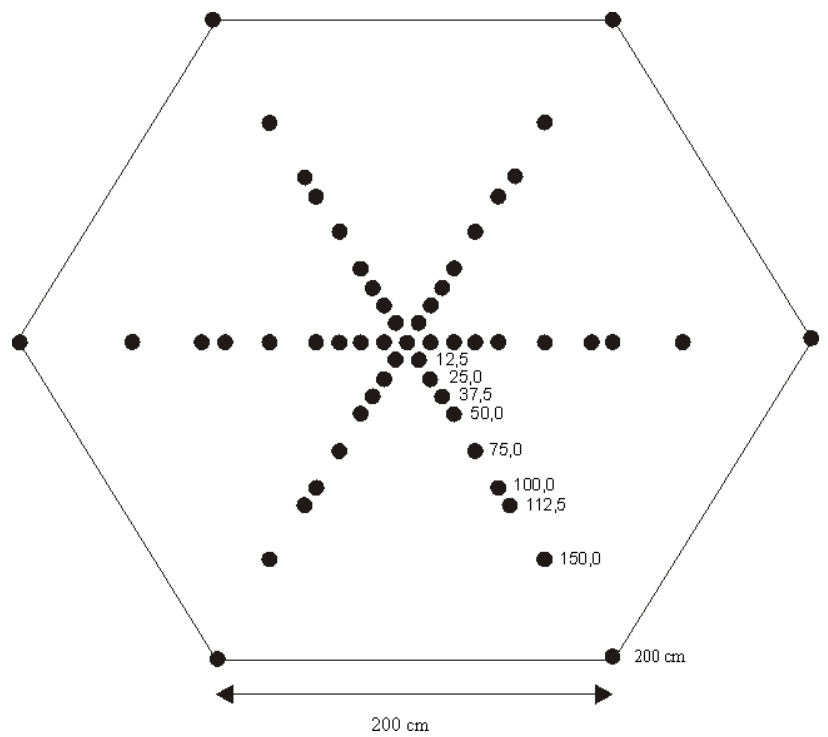

Figura 1. Hexágono de amostragem, com dez amostras simples por semidiagonal, sendo a amostra simples central comum às seis semidiagonais.

são numericamente múltiplos desta, foram idealizados modelos de possíveis indivíduos-solo em forma de prismas hexagonais (10 $\mathrm{cm}$ de profundidade), formados por um conjunto de pequenas colunas primordiais em forma de prismas hexagonais de $4,167 \mathrm{~cm}$ de lado $\left(45,1 \mathrm{~cm}^{2}\right.$ de área por $10 \mathrm{~cm}$ de profundidade $=$ $\left.0,451 \mathrm{dm}^{3}\right)$, correspondentes a 1/3 da distância inicial de amostragem (12,5 cm) (Figura 2).

Partindo dos modelos idealizados para os possíveis indivíduos-solo, em forma de prismas hexagonais, as amostras simples foram agrupadas a partir do centro do hexágono de amostragem, segundo as áreas que representavam (considerando a profundidade de amostragem de $10 \mathrm{~cm}$ ), gerando nove grupos com diferentes números de amostras simples, relativos a nove indivíduos-solo distintos em forma de prismas hexagonais (Quadro 1).

As amostras simples foram secas ao ar, peneiradas ( $2 \mathrm{~mm})$, homogeneizadas e analisadas individualmente, sendo determinados: $\mathrm{pH}$ em água $(1: 2,5)$, teores de $\mathrm{P}$ e $\mathrm{K}$ disponíveis (Mehlich-1) e de $\mathrm{Ca}^{2+} \mathrm{e} \mathrm{Mg}^{2+}(\mathrm{KCl}$ $\left.1 \mathrm{~mol} \mathrm{~L}^{-1}\right), \mathrm{H}+\mathrm{Al}\left(\mathrm{Ca}(\mathrm{OAc})_{2}, 0,5 \mathrm{~mol} \mathrm{~L}^{-1} . \mathrm{pH} 7\right.$; Defelipo \& Ribeiro, 1996), P-remanescente (P em solução após agitação por $1 \mathrm{~h}$ com $60 \mathrm{mg} \mathrm{L}^{-1} \mathrm{de} \mathrm{P} \mathrm{em}$ $\mathrm{CaCl}_{2}$ 0,01 mol L-1, na relação solo:solução de 1:10, Alvarez V. et al., 2000) e carbono orgânico (Método Walkley-Black; Jackson, 1976).

Os dados foram analisados como em um delineamento de blocos casualizados, com cinco repetições (cinco hexágonos por situação de manejo-coleta).

Foi realizada a análise de variância por situação de manejo-coleta (Unidades de amostra, $8 \mathrm{GL}$; Hexágonos de amostragem, 4 GL; Resíduo, 32 GL). 
Quadro 1. Unidades de amostra avaliadas, distâncias a partir do centro do hexágono de amostragem, número de amostras simples pertinente a cada unidade de amostra, número de colunas primordiais representadas e área efetiva dos possíveis indivíduos-solo em forma de prismas hexagonais (unidades de amostra)

\begin{tabular}{|c|c|c|c|c|}
\hline $\begin{array}{l}\text { Unidade de } \\
\text { amostra }\end{array}$ & $\begin{array}{c}\text { Distância a partir do centro } \\
\text { do hexágono }\end{array}$ & $\begin{array}{l}\text { № amostras } \\
\text { simples }\end{array}$ & $\begin{array}{l}\text { № de colunas } \\
\text { primordial }^{(3)}\end{array}$ & $\begin{array}{l}\text { Área efetiva do } \\
\text { indivíduo-solo }\end{array}$ \\
\hline & $\mathrm{cm}$ & & & $\mathrm{m}^{2}$ \\
\hline A & Até $\quad 12,5^{(1)}(18,75)^{(2)}$ & 7 & 20,25 & 0,091 \\
\hline $\mathrm{B}$ & Até $\quad 25,0 \quad(31,25)$ & 13 & 56,25 & 0,254 \\
\hline $\mathrm{C}$ & Até $\quad 37,5 \quad(43,75)$ & 19 & 110,25 & 0,497 \\
\hline $\mathrm{D}$ & Até $\quad 50,0 \quad(56,25)$ & 25 & 182,25 & 0,822 \\
\hline $\mathrm{E}$ & Até $\quad 75,0 \quad(81,25)$ & 31 & 380,25 & 1,715 \\
\hline $\mathrm{F}$ & Até $100,0 \quad(106,25)$ & 37 & 650,25 & 2,933 \\
\hline $\mathrm{G}$ & Até $112,5 \quad(118,75)$ & 43 & 812,25 & 3,664 \\
\hline $\mathrm{H}$ & Até $150,0 \quad(156,25)$ & 49 & $1.406,25$ & 6,343 \\
\hline I & Até $200,0 \quad(206,25)$ & 55 & $2.450,25$ & 11,052 \\
\hline
\end{tabular}

(1) Medida a partir do centro do orifício de coleta das amostras simples. ${ }^{(2)}$ Comprimento da semidiagonal do indivíduo solo representado. ${ }^{(3)}$ Colunas em forma de prismas hexagonais $(4,167 \mathrm{~cm}$ de lado por $10 \mathrm{~cm}$ de profundidade).

Utilizando a análise de regressão (modelo linearplatô ou quadrático-platô), foi determinada a dimensão (área) que representaria o indivíduo solo, para uma profundidade de $10 \mathrm{~cm}$. A dimensão da unidade de amostra de solo foi definida no ponto onde se iniciava a formação do platô no modelo, observando-se que, quanto maior a variabilidade a curta distância no solo, menor a dimensão da unidade de amostra de solo.

A partir destes resultados, foi determinado o número de amostras simples necessário à representação do indivíduo solo (unidade de amostra).

\section{RESULTADOS E DISCUSSÃO}

O indivíduo solo, em forma de prisma hexagonal, foi dimensionado por meio de equações de regressão. Foram selecionados, para isso, os modelos linear-platô ou quadrático-platô, com base no comportamento das médias das características químicas avaliadas e das médias dos desvios-padrão destas características (Quadros 2 e 3), de acordo com a dimensão das unidades de amostra de solo, na significância dos coeficientes de regressão (limite máximo de 0,40) e nos valores de $\mathrm{R}^{2}$ (Alvarez V. \& Alvarez, 2003), para as três situações de manejo-coleta (PD, PCAA e PCDA).

A dimensão do indivíduo solo variou conforme o atributo da fertilidade do solo avaliada e com o tipo de situação de manejo-coleta (PD, PCAA e PCDA). A dimensão que caracterizou a fertilidade local média de determinada característica foi, de maneira geral, menor do que a dimensão que caracterizou a estimativa da variabilidade dessa mesma característica (Quadros 4 e 5). Esse resultado pode ser explicado com base no modelo para o indivíduo solo em forma de prisma hexagonal (Figura 2), uma vez que diferentes grupos de colunas primordiais podem apresentar a mesma média de determinada característica, mas com distintas variabilidades. Dessa forma, pressupõe-se que a dimensão que melhor caracterize a variabilidade seja mais importante para a definição da dimensão "ideal" da unidade de amostra do que a que caracterize a média local.

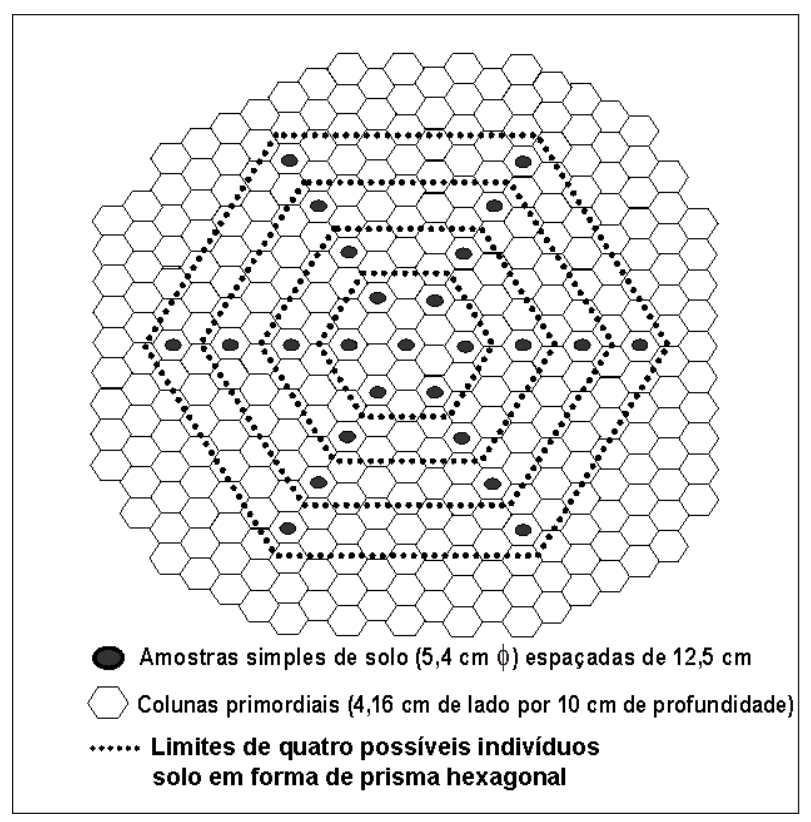

Figura 2. Modelo hipotético de quatro possíveis indivíduos-solo (unidades de amostra) em forma de prismas hexagonais, formados pelas respectivas colunas primordiais em forma de prismas hexagonais $(4,167 \mathrm{~cm}$ de lado por $10 \mathrm{~cm})$. 


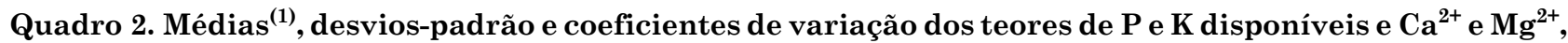
determinados em indivíduos-solo em forma de prismas hexagonais, em três situações de manejo-coleta ${ }^{(2)}$

\begin{tabular}{|c|c|c|c|c|c|c|c|c|c|}
\hline \multirow{3}{*}{$\begin{array}{l}\text { Dimensão do } \\
\text { indivíduo solo }\end{array}$} & \multicolumn{9}{|c|}{ Situação de manejo-coleta } \\
\hline & \multicolumn{3}{|c|}{ PD } & \multicolumn{3}{|c|}{ PCAA } & \multicolumn{3}{|c|}{ PCDA } \\
\hline & $\overline{\boldsymbol{y}}_{\mathrm{ij}}$ & $\overline{\boldsymbol{S}}_{\mathrm{ij}}$ & $\overline{C V}$ ij & $\bar{y}_{i j}$ & $\overline{\boldsymbol{S}}_{\mathrm{ij}}$ & $\overline{C V}_{\mathrm{ij}}$ & $\overline{\bar{y}_{\mathrm{ij}}}$ & $\overline{\boldsymbol{S}}_{\mathrm{ij}}$ & $\overline{C V}$ ij \\
\hline & \multicolumn{2}{|c|}{$-\mathrm{mg} \mathrm{dm}^{-3}-$} & $\%$ & \multicolumn{2}{|c|}{$-\mathrm{mg} \mathrm{dm}^{-3}-$} & $\%$ & \multicolumn{2}{|c|}{$-\mathrm{mg} \mathrm{dm}^{-3} \ldots$} & $\%$ \\
\hline & & & & & $\mathrm{P}$ & & & & \\
\hline $0,091 \times 0,1$ & 61,5 & 33,88 & 61,28 & 27,2 & 2,98 & 10,64 & 35,4 & 2,36 & 6,68 \\
\hline $0,254 \times 0,1$ & 58,1 & 31,59 & 61,96 & 28,2 & 4,76 & 15,66 & 35,4 & 2,12 & 6,00 \\
\hline $0,497 \times 0,1$ & 54,8 & 27,79 & 58,78 & 29,3 & 6,90 & 21,99 & 35,6 & 2,21 & 6,21 \\
\hline $0,822 \times 0,1$ & 55,0 & 30,84 & 65,19 & 31,8 & 14,63 & 50,29 & 35,6 & 2,46 & 6,90 \\
\hline $1,715 \times 0,1$ & 55,1 & 34,20 & 69,49 & 31,5 & 13,93 & 48,61 & 35,7 & 2,60 & 7,30 \\
\hline $2,933 \times 0,1$ & 55,6 & 34,35 & 69,84 & 31,7 & 14,25 & 48,85 & 35,6 & 2,62 & 7,37 \\
\hline $3,664 \times 0,1$ & 55,2 & 33,29 & 68,08 & 31,8 & 13,59 & 45,79 & 35,7 & 2,66 & 7,45 \\
\hline $6,343 \times 0,1$ & 55,5 & 32,95 & 66,61 & 31,8 & 13,00 & 43,83 & 35,6 & 2,65 & 7,42 \\
\hline \multirow[t]{2}{*}{$11,052 \times 0,1$} & 56,1 & 34,16 & 67,89 & 31,7 & 12,78 & 42,99 & 35,6 & 2,87 & 8,01 \\
\hline & \multicolumn{9}{|c|}{$\mathrm{K}$} \\
\hline $0,091 \times 0,1$ & 285 & 54,84 & 19,99 & 180 & 32,79 & 17,98 & 158 & 21,26 & 13,77 \\
\hline $0,254 \times 0,1$ & 287 & 51,38 & 18,64 & 177 & 35,54 & 20,20 & 156 & 21,49 & 13,86 \\
\hline $0,497 \times 0,1$ & 286 & 54,24 & 19,55 & 177 & 40,59 & 23,38 & 155 & 23,90 & 15,36 \\
\hline $0,822 \times 0,1$ & 286 & 51,86 & 18,51 & 176 & 41,73 & 24,18 & 155 & 26,01 & 16,77 \\
\hline $1,715 \times 0,1$ & 289 & 53,03 & 18,66 & 173 & 42,44 & 24,87 & 158 & 28,04 & 17,69 \\
\hline $2,933 \times 0,1$ & 288 & 52,42 & 18,43 & 171 & 40,55 & 23,90 & 160 & 28,69 & 17,85 \\
\hline $3,664 \times 0,1$ & 287 & 53,29 & 18,72 & 171 & 40,05 & 23,55 & 160 & 28,71 & 17,80 \\
\hline $6,343 \times 0,1$ & 287 & 52,54 & 18,40 & 173 & 41,32 & 24,01 & 159 & 29,96 & 18,66 \\
\hline \multirow[t]{3}{*}{$11,052 \times 0,1$} & 285 & 53,60 & 18,95 & 173 & 41,78 & 24,20 & 160 & 30,51 & 18,94 \\
\hline & & & & & $\mathrm{Ca}^{2+}$ & & & & \\
\hline & \multicolumn{2}{|c|}{$-\mathrm{cmol}_{\mathrm{c}} \mathrm{dm}^{-3}-$} & $\%$ & \multicolumn{2}{|c|}{$-\mathrm{cmol}_{\mathrm{c}} \mathrm{dm}^{-3}-$} & $\%$ & \multicolumn{2}{|c|}{$-\mathrm{cmol}_{\mathrm{c}} \mathrm{dm}^{-3}-$} & $\%$ \\
\hline $0,091 \times 0,1$ & 6,55 & 0,46 & 7,18 & 3,67 & 0,24 & 6,52 & 3,99 & 0,27 & 6,55 \\
\hline $0,254 \times 0,1$ & 6,57 & 0,47 & 7,35 & 3,64 & 0,24 & 6,52 & 4,02 & 0,25 & 6,12 \\
\hline $0,497 \times 0,1$ & 6,58 & 0,47 & 7,23 & 3,63 & 0,26 & 7,07 & 4,03 & 0,25 & 6,13 \\
\hline $0,822 \times 0,1$ & 6,58 & 0,47 & 7,26 & 3,65 & 0,27 & 7,32 & 4,04 & 0,26 & 6,33 \\
\hline $1,715 \times 0,1$ & 6,60 & 0,47 & 7,25 & 3,65 & 0,27 & 7,34 & 4,05 & 0,26 & 6,35 \\
\hline $2,933 \times 0,1$ & 6,61 & 0,48 & 7,45 & 3,65 & 0,26 & 7,13 & 4,05 & 0,26 & 6,33 \\
\hline $3,664 \times 0,1$ & 6,63 & 0,51 & 7,94 & 3,65 & 0,26 & 7,12 & 4,05 & 0,26 & 6,35 \\
\hline $6,343 \times 0,1$ & 6,64 & 0,54 & 8,31 & 3,66 & 0,26 & 7,20 & 4,05 & 0,26 & 6,37 \\
\hline \multirow[t]{2}{*}{$11,052 \times 0,1$} & 6,65 & 0,60 & 9,14 & 3,65 & 0,28 & 7,58 & 4,05 & 0,26 & 6,23 \\
\hline & & & & & $\mathrm{Mg}^{2+}$ & & & & \\
\hline $0,091 \times 0,1$ & 1,57 & 0,10 & 6,77 & 0,60 & 0,07 & 10,71 & 0,77 & 0,03 & 3,34 \\
\hline $0,254 \times 0,1$ & 1,59 & 0,13 & 8,60 & 0,59 & 0,07 & 11,44 & 0,78 & 0,03 & 3,73 \\
\hline $0,497 \times 0,1$ & 1,61 & 0,14 & 8,84 & 0,58 & 0,07 & 12,54 & 0,78 & 0,04 & 4,58 \\
\hline $0,822 \times 0,1$ & 1,61 & 0,14 & 9,05 & 0,58 & 0,07 & 12,26 & 0,78 & 0,04 & 5,06 \\
\hline $1,715 \times 0,1$ & 1,62 & 0,15 & 9,25 & 0,58 & 0,07 & 12,56 & 0,78 & 0,04 & 5,11 \\
\hline $2,933 \times 0,1$ & 1,62 & 0,16 & 10,26 & 0,58 & 0,07 & 12,41 & 0,78 & 0,04 & 5,09 \\
\hline $3,664 \times 0,1$ & 1,62 & 0,17 & 10,64 & 0,58 & 0,08 & 13,89 & 0,78 & 0,04 & 5,27 \\
\hline $6,343 \times 0,1$ & 1,62 & 0,18 & 11,29 & 0,58 & 0,08 & 14,21 & 0,78 & 0,04 & 5,42 \\
\hline $11,052 \times 0,1$ & 1,61 & 0,20 & 12,51 & 0,58 & 0,08 & 14,47 & 0,78 & 0,04 & 5,52 \\
\hline
\end{tabular}

(1) Médias de cinco possíveis indivíduos (cinco hexágonos de amostragem) por situação de manejo-coleta. ${ }^{(2)} \mathrm{PD}=\mathrm{Plantio}$ direto; PCAA = Plantio convencional antes da aração; PCDA = Plantio convencional depois da aração.

Para P no PD, a coleta de amostras simples $(10 \mathrm{~cm}$ de profundidade) numa área hexagonal de $0,386 \mathrm{~m}^{2}$ caracteriza o indivíduo solo $\left(0,386 \mathrm{~m}^{2} \times 0,1 \mathrm{~m}\right)$, em relação à disponibilidade local média de $\mathrm{P}$ (Quadro 4). Como o PD constava de uma rotação milho - feijão com sete anos a partir do plantio, pode-se esperar que esta dimensão $\left(0,386 \mathrm{~m}^{2} \times 0,1 \mathrm{~m}\right)$ seja capaz de representar a disponibilidade local média de $\mathrm{P}$, uma vez que há elevado efeito residual do adubo aplicado nos cultivos anteriores nas linhas de plantio. Por outro lado, para que as mesovariações $(0,05$ a $2 \mathrm{~m})$ sejam incluídas no volume de solo representativo do indivíduo, a unidade de amostra de solo deve apresentar a dimensão de $0,949 \mathrm{~m}^{2}$ x 0,1 m (Quadro 4). 
Quadro 3. Médias ${ }^{(1)}$, desvios-padrão e coeficientes de variação de H + Al, matéria orgânica (MO), P-rem e pH, determinados em indivíduos-solo em forma de prismas hexagonais, em três situações de manejo-coleta ${ }^{(2)}$

\begin{tabular}{|c|c|c|c|c|c|c|c|c|c|}
\hline \multirow{3}{*}{$\begin{array}{l}\text { Dimensão do } \\
\text { indivíduo solo }\end{array}$} & \multicolumn{9}{|c|}{ Situação de manejo-coleta } \\
\hline & \multicolumn{3}{|c|}{ PD } & \multicolumn{3}{|c|}{ PCAA } & \multicolumn{3}{|c|}{ PCDA } \\
\hline & $\overline{\boldsymbol{y}}_{\mathrm{ij}}$ & $\overline{\boldsymbol{S}}_{\mathrm{ij}}$ & $\overline{C V}$ ij & $\bar{y}_{\mathrm{ij}}$ & $\overline{\boldsymbol{S}}_{\mathrm{ij}}$ & $\overline{C V}$ ij & $\bar{y}_{\mathrm{ij}}$ & $\overline{\boldsymbol{S}}_{\mathrm{ij}}$ & $\overline{C V} \mathrm{ij}$ \\
\hline & \multicolumn{9}{|c|}{$\mathrm{H}+\mathrm{Al}$} \\
\hline & \multicolumn{2}{|c|}{$-\mathrm{cmol}_{\mathrm{c}} \mathrm{dm}^{-3}-$} & $\%$ & \multicolumn{2}{|c|}{$-\mathrm{cmol}_{\mathrm{c}} \mathrm{dm}^{-3}-$} & $\%$ & \multicolumn{2}{|c|}{$-\mathrm{cmol}_{\mathrm{c}} \mathrm{dm}^{-3}-$} & $\%$ \\
\hline $0,091 \times 0,1$ & 3,10 & 0,39 & 12,74 & 6,38 & 0,42 & 6,63 & 3,78 & 0,25 & 6,59 \\
\hline $0,254 \times 0,1$ & 3,05 & 0,40 & 13,76 & 6,54 & 0,53 & 8,11 & 3,81 & 0,25 & 6,72 \\
\hline $0,497 \times 0,1$ & 3,05 & 0,40 & 14,18 & 6,57 & 0,61 & 9,46 & 3,85 & 0,26 & 6,75 \\
\hline $0,822 \times 0,1$ & 3,02 & 0,40 & 14,45 & 6,64 & 0,63 & 9,56 & 3,88 & 0,27 & 7,01 \\
\hline $1,715 \times 0,1$ & 3,02 & 0,41 & 14,79 & 6,68 & 0,68 & 10,29 & 3,91 & 0,27 & 6,87 \\
\hline $2,933 \times 0,1$ & 3,01 & 0,42 & 15,15 & 6,72 & 0,70 & 10,45 & 3,91 & 0,27 & 6,82 \\
\hline $3,664 \times 0,1$ & 3,01 & 0,45 & 16,22 & 6,74 & 0,68 & 10,18 & 3,92 & 0,26 & 6,74 \\
\hline $6,343 \times 0,1$ & 3,01 & 0,48 & 17,65 & 6,72 & 0,70 & 10,55 & 3,91 & 0,26 & 6,75 \\
\hline \multirow[t]{3}{*}{$11,052 \times 0,1$} & 3,03 & 0,53 & 19,52 & 6,73 & 0,73 & 10,98 & 3,91 & 0,26 & 6,76 \\
\hline & \multicolumn{9}{|c|}{ MO } \\
\hline & \multicolumn{2}{|c|}{ - dag kg.1 } & $\%$ & \multicolumn{2}{|c|}{ - dag kg-1 _- } & $\%$ & \multicolumn{2}{|c|}{ - dag kg-1 } & $\%$ \\
\hline $0,091 \times 0,1$ & 4,35 & 0,66 & 15,36 & 3,66 & 0,49 & 13,39 & 2,85 & 0,54 & 18,90 \\
\hline $0,254 \times 0,1$ & 4,26 & 0,64 & 15,19 & 3,69 & 0,49 & 13,27 & 2,76 & 0,53 & 19,49 \\
\hline $0,497 \times 0,1$ & 4,27 & 0,63 & 14,96 & 3,71 & 0,52 & 13,98 & 2,74 & 0,52 & 19,29 \\
\hline $0,822 \times 0,1$ & 4,26 & 0,60 & 14,32 & 3,70 & 0,52 & 14,09 & 2,78 & 0,55 & 19,91 \\
\hline $1,715 \times 0,1$ & 4,27 & 0,59 & 13,89 & 3,70 & 0,51 & 13,96 & 2,77 & 0,52 & 19,09 \\
\hline $2,933 \times 0,1$ & 4,30 & 0,59 & 13,95 & 3,69 & 0,50 & 13,51 & 2,77 & 0,51 & 18,69 \\
\hline $3,664 \times 0,1$ & 4,33 & 0,62 & 14,62 & 3,68 & 0,50 & 13,51 & 2,77 & 0,50 & 18,42 \\
\hline $6,343 \times 0,1$ & 4,36 & 0,64 & 14,89 & 3,68 & 0,50 & 13,67 & 2,76 & 0,49 & 18,08 \\
\hline \multirow[t]{3}{*}{$11,052 \times 0,1$} & 4,35 & 0,64 & 14,96 & 3,67 & 0,49 & 13,47 & 2,76 & 0,49 & 18,06 \\
\hline & \multicolumn{9}{|c|}{ P-rem } \\
\hline & \multicolumn{2}{|c|}{$\mathrm{mg} \mathrm{L}^{-1}$} & $\%$ & \multicolumn{2}{|c|}{$\mathrm{mg} \mathrm{L}^{-1}$} & $\%$ & \multicolumn{2}{|c|}{$\mathrm{mg} \mathrm{L}^{-1}$} & $\%$ \\
\hline $0,091 \times 0,1$ & 28,00 & 1,62 & 5,84 & 23,93 & 1,27 & 5,35 & 22,27 & 0,87 & 3,91 \\
\hline $0,254 \times 0,1$ & 27,91 & 1,63 & 5,85 & 23,92 & 1,28 & 5,42 & 22,38 & 0,92 & 4,12 \\
\hline $0,497 \times 0,1$ & 27,89 & 1,56 & 5,59 & 23,96 & 1,45 & 6,10 & 22,45 & 0,93 & 4,16 \\
\hline $0,822 \times 0,1$ & 27,90 & 1,62 & 5,82 & 24,07 & 1,47 & 6,19 & 22,54 & 0,96 & 4,25 \\
\hline $1,715 \times 0,1$ & 27,91 & 1,74 & 6,25 & 24,01 & 1,48 & 6,21 & 22,60 & 0,98 & 4,34 \\
\hline $2,933 \times 0,1$ & 27,97 & 1,89 & 6,74 & 23,94 & 1,55 & 6,53 & 22,63 & 0,99 & 4,35 \\
\hline $3,664 \times 0,1$ & 27,95 & 1,93 & 6,92 & 23,95 & 1,52 & 6,37 & 22,65 & 1,02 & 4,50 \\
\hline $6,343 \times 0,1$ & 27,96 & 1,94 & 6,94 & 23,96 & 1,54 & 6,43 & 22,63 & 1,05 & 4,62 \\
\hline \multirow[t]{2}{*}{$11,052 \times 0,1$} & 27,93 & 2,00 & 7,19 & 23,94 & 1,54 & 6,46 & 22,59 & 1,07 & 4,73 \\
\hline & \multicolumn{9}{|c|}{$\mathrm{pH}$} \\
\hline $0,091 \times 0,1$ & 6,31 & 0,14 & 2,27 & 5,03 & 0,17 & 3,35 & 5,61 & 0,07 & 1,20 \\
\hline $0,254 \times 0,1$ & 6,34 & 0,15 & 2,38 & 5,02 & 0,20 & 3,98 & 5,62 & 0,06 & 1,05 \\
\hline $0,497 \times 0,1$ & 6,36 & 0,16 & 2,47 & 5,02 & 0,24 & 4,77 & 5,62 & 0,07 & 1,19 \\
\hline $0,822 \times 0,1$ & 6,37 & 0,16 & 2,50 & 5,02 & 0,25 & 4,91 & 5,62 & 0,08 & 1,35 \\
\hline $1,715 \times 0,1$ & 6,38 & 0,16 & 2,52 & 5,00 & 0,28 & 5,59 & 5,62 & 0,07 & 1,29 \\
\hline $2,933 \times 0,1$ & 6,38 & 0,17 & 2,70 & 4,99 & 0,28 & 5,67 & 5,62 & 0,07 & 1,27 \\
\hline $3,664 \times 0,1$ & 6,39 & 0,18 & 2,80 & 4,99 & 0,28 & 5,62 & 5,62 & 0,07 & 1,24 \\
\hline $6,343 \times 0,1$ & 6,39 & 0,18 & 2,88 & 5,00 & 0,28 & 5,66 & 5,61 & 0,07 & 1,33 \\
\hline $11,052 \times 0,1$ & 6,38 & 0,21 & 3,24 & 4,99 & 0,30 & 6,00 & 5,62 & 0,07 & 1,30 \\
\hline
\end{tabular}

(1) Médias de cinco possíveis indivíduos (cinco hexágonos de amostragem) por situação de manejo-coleta. ${ }^{(2)}$ PD = Plantio direto; PCAA = Plantio convencional antes da aração; PCDA = Plantio convencional depois da aração.

A unidade de amostra de solo não deve contemplar apenas a fertilidade local média, mas também as variações representativas a curtas distâncias na composição do solo. Assim, admite-se que a unidade de amostra de solo para esta situação (P-PD) seja mais bem representada por um volume de $0,949 \mathrm{~m}^{2}$ x $0,1 \mathrm{~m}$.
Com esta dimensão, contemplam-se as variações a curtas distâncias no solo, além de caracterizar a disponibilidade de $\mathrm{P}$ de forma tão ou mais eficiente do que a dimensão de $0,386 \mathrm{~m}^{2} \times 0,1 \mathrm{~m}$, uma vez que, com aquela, ultrapassa-se o início do platô para o teor médio de $\mathrm{P}$ (Quadro 4). 
Para P no PCAA, a dimensão do indivíduo foi de $1,200 \mathrm{~m}^{2} \times 0,1 \mathrm{~m}$, com base no desvio-padrão, ficando a dimensão para caracterizar a fertilidade local média muito próxima desta (Quadro 4). Nessa condição, como apenas os sulcos de plantio da última cultura estão presentes no momento de coleta, o indivíduo solo deve contemplar a fertilidade média entre os pontos de maior (sulco) e menor concentração (entrelinha), bem como a variabilidade gerada pelos próprios sulcos.

Quadro 4. Equações de regressão das médias ${ }^{(1)}$ dos teores de P e K disponível e Ca e Mg trocáveis e das

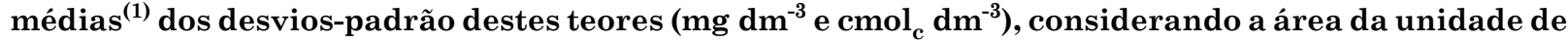
amostra de solo e área dimensionada para a unidade de amostra em forma de prisma hexagonal, para uma profundidade de $10 \mathrm{~cm}$, em três situações de manejo-coleta

\begin{tabular}{|c|c|c|c|c|}
\hline Sit uação(2) & \multicolumn{2}{|c|}{ Equaçôes de regressão } & $\mathbf{R}^{2}$ & Área $\left(\mathrm{m}^{2}\right)$ \\
\hline \multicolumn{5}{|c|}{$\mathrm{P}$ disponível } \\
\hline $\mathrm{PD}$ & $\begin{array}{l}\hat{y}=63,397-20,911^{(0,168)} \mathrm{a} \\
\hat{y}=\mathrm{b}_{2}=55,328\end{array}$ & $\begin{array}{l}\forall 0,091 \leq \mathrm{a} \leq 0,386 \mathrm{~m}^{2} \\
\forall 0,386 \leq \mathrm{a} \leq 11,052 \mathrm{~m}^{2}\end{array}$ & 0,971 & 0,386 \\
\hline & $\begin{array}{l}\hat{s}=36,939-31,923^{(0,150)} \mathrm{a}+29,600^{(0,203)} \mathrm{a}^{2} \\
\hat{s}=\mathrm{b}_{2}=33,299\end{array}$ & $\begin{array}{l}\forall 0,091 \leq \mathrm{a} \leq 0,949 \mathrm{~m}^{2} \\
\forall 0,949 \leq \mathrm{a} \leq 11,052 \mathrm{~m}^{2}\end{array}$ & 0,980 & 0,949 \\
\hline PCAA & $\begin{array}{l}\hat{y}=26,769+5,246^{* 2} \mathrm{a} \\
\hat{y}=\mathrm{b}_{2}=31,704\end{array}$ & $\begin{array}{l}\forall 0,091 \leq \mathrm{a} \leq 0,941 \mathrm{~m}^{2} \\
\forall 0,941 \leq \mathrm{a} \leq 11,052 \mathrm{~m}^{2}\end{array}$ & 0,996 & 0,941 \\
\hline & $\begin{array}{l}\hat{s}=2,19+9,592^{*} \mathrm{a} \\
\hat{s}=\mathrm{b}_{2}=13,696\end{array}$ & $\begin{array}{l}\forall 0,091 \leq \mathrm{a} \leq 1,200 \mathrm{~m}^{2} \\
\forall 1,200 \leq \mathrm{a} \leq 11,052 \mathrm{~m}^{2}\end{array}$ & 0,984 & 1,200 \\
\hline PCDA & $\begin{array}{l}\hat{y}=35,376+0,211^{(0,155)} \mathrm{a} \\
\hat{y}=\mathrm{b}_{2}=35,631\end{array}$ & $\begin{array}{l}\forall 0,091 \leq \mathrm{a} \leq 1,209 \mathrm{~m}^{2} \\
\forall 1,209 \leq \mathrm{a} \leq 11,052 \mathrm{~m}^{2}\end{array}$ & 0,998 & 1,209 \\
\hline & $\hat{s}=\bar{s}=2,506$ & $\forall 0,091 \leq \mathrm{a} \leq 11,052 \mathrm{~m}^{2}$ & & 0,091 \\
\hline \multicolumn{5}{|c|}{$\mathrm{K}$ disponível } \\
\hline $\mathrm{PD}$ & $\hat{y}=\bar{y}=286,667$ & $\forall 0,091 \leq \mathrm{a} \leq 11,052 \mathrm{~m}^{2}$ & & 0,091 \\
\hline & $\hat{s}=\bar{s}=53,022$ & $\forall 0,091 \leq \mathrm{a} \leq 11,052 \mathrm{~m}^{2}$ & & 0,091 \\
\hline PCAA & $\begin{array}{l}\hat{y}=179,469-3,990^{(0,202)} \mathrm{a} \\
\hat{y}=\mathrm{b}_{2}=172,063\end{array}$ & $\begin{array}{l}\forall 0,091 \leq \mathrm{a} \leq 1,856 \mathrm{~m}^{2} \\
\forall 1,856 \leq \mathrm{a} \leq 11,052 \mathrm{~m}^{2}\end{array}$ & 0,940 & 1,856 \\
\hline & $\begin{array}{l}\hat{s}=30,879+19,329^{*} \mathrm{a} \\
\hat{s}=\mathrm{b}_{2}=41,315\end{array}$ & $\begin{array}{l}\forall 0,091 \leq \mathrm{a} \leq 0,540 \mathrm{~m}^{2} \\
\forall 0,540 \leq \mathrm{a} \leq 11,052 \mathrm{~m}^{2}\end{array}$ & 0,954 & 0,540 \\
\hline PCDA & $\begin{array}{l}\hat{y}=158,120-7,661^{(0,378)} \mathrm{a}+4,878^{(0,288)} \mathrm{a}^{2} \\
\hat{y}=\mathrm{b}_{2}=159,301\end{array}$ & $\begin{array}{l}\forall 0,091 \leq \mathrm{a} \leq 1,712 \mathrm{~m}^{2} \\
\forall 1,712 \leq \mathrm{a} \leq 11,052 \mathrm{~m}^{2}\end{array}$ & 0,911 & 1,712 \\
\hline & $\begin{array}{l}\hat{s}=20,279+6,940^{* *} \mathrm{a} \\
\hat{s}=\mathrm{b}_{2}=29,181\end{array}$ & $\begin{array}{l}\forall 0,091 \leq \mathrm{a} \leq 1,283 \mathrm{~m}^{2} \\
\forall 1,283 \leq \mathrm{a} \leq 11,052 \mathrm{~m}^{2}\end{array}$ & 0,954 & 1,283 \\
\hline \multicolumn{5}{|c|}{ Ca trocável } \\
\hline PD & $\begin{array}{l}\hat{y}=6,565+0,018^{*} \mathrm{a} \\
\hat{y}=\mathrm{b}_{2}=6,646\end{array}$ & $\begin{array}{l}\forall 0,091 \leq \mathrm{a} \leq 4,421 \mathrm{~m}^{2} \\
\forall 4,421 \leq \mathrm{a} \leq 11,052 \mathrm{~m}^{2}\end{array}$ & 0,904 & 4,421 \\
\hline & $\begin{array}{l}\hat{s}=0,460+0,012^{\circ} \mathrm{a} \\
\hat{y}=3,680-0,205^{(0,208)} \mathrm{a}+0,201^{(0,239)} \mathbf{a}^{2}\end{array}$ & $\begin{array}{l}\forall 0,091 \leq \mathrm{a} \leq 11,052 \mathrm{~m}^{2} \\
\forall 0,091 \leq \mathrm{a} \leq 0,862 \mathrm{~m}^{2}\end{array}$ & 0,969 & 11,052 \\
\hline PCAA & $\hat{y}=\mathrm{b}_{2}=3,652$ & $\forall 0,862 \leq \mathrm{a} \leq 11,052 \mathrm{~m}^{2}$ & 0,996 & 0,862 \\
\hline \multirow{3}{*}{ PCDA } & $\begin{array}{l}\hat{s}=0,229+0,049^{(0,221)} \mathrm{a} \\
\hat{s}=\mathrm{b}_{2}=0,265\end{array}$ & $\begin{array}{l}\forall 0,091 \leq \mathrm{a} \leq 0,738 \mathrm{~m}^{2} \\
\forall 0,738 \leq \mathrm{a} \leq 11,052 \mathrm{~m}^{2}\end{array}$ & 0,825 & 0,738 \\
\hline & $\begin{array}{l}\hat{y}=3,985+0,101^{*} \mathrm{a} \\
\hat{y}=\mathrm{b}_{2}=4,050\end{array}$ & $\begin{array}{l}\forall 0,091 \leq \mathrm{a} \leq 0,645 \mathrm{~m}^{2} \\
\forall 0,645 \leq \mathrm{a} \leq 11,052 \mathrm{~m}^{2}\end{array}$ & 0,955 & 0,645 \\
\hline & $\begin{array}{l}\hat{s}=0,265-0,098^{(0,232)} \mathrm{a}+0,102^{(0,236)} \mathrm{a}^{2} \\
\hat{s}=\mathrm{b}_{2}=0,260\end{array}$ & $\begin{array}{l}\forall 0,091 \leq \mathrm{a} \leq 0,814 \mathrm{~m}^{2} \\
\forall 0,814 \leq \mathrm{a} \leq 11,052 \mathrm{~m}^{2}\end{array}$ & 0,908 & 0,814 \\
\hline
\end{tabular}


Quadro 4. Continuação

\begin{tabular}{|c|c|c|c|c|}
\hline Situação(2) & \multicolumn{2}{|c|}{ Equações de regressão } & $\mathbf{R}^{2}$ & Área $\left(\mathrm{m}^{2}\right)$ \\
\hline \multicolumn{5}{|c|}{ Mg trocável } \\
\hline \multirow{4}{*}{ PD } & $\hat{y}=1,567+0,088^{\circ} a$ & $\forall 0,091 \leq \mathrm{a} \leq 0,576 \mathrm{~m}^{2}$ & & \\
\hline & $\hat{y}=\mathrm{b}_{2}=1,614$ & $\forall 0,576 \leq \mathrm{a} \leq 11,052 \mathrm{~m}^{2}$ & 0,889 & 0,576 \\
\hline & $\hat{s}=0,125+0,013^{* *} \mathrm{a}$ & $\forall 0,091 \leq \mathrm{a} \leq 4,774 \mathrm{~m}^{2}$ & 0.862 & 4.774 \\
\hline & $\hat{s}=\mathrm{b}_{2}=0,188$ & $\forall 4,774 \leq \mathrm{a} \leq 11,052 \mathrm{~m}^{2}$ & 0,862 & 4,774 \\
\hline PCAA & $\hat{y}=\bar{y}=0,583$ & $\forall 0,091 \leq \mathrm{a} \leq 11,052 \mathrm{~m}^{2}$ & & 0,091 \\
\hline \multirow{3}{*}{ PCDA } & $\begin{array}{l}\hat{s}=0,069+0,001^{(0,359)} \mathrm{a} \\
\hat{s}=\mathrm{b}_{2}=0,084\end{array}$ & $\begin{array}{l}\forall 0,091 \leq \mathrm{a} \leq 10,50 \mathrm{~m}^{2} \\
\forall 10,50 \leq \mathrm{a} \leq 11,052 \mathrm{~m}^{2}\end{array}$ & 0,949 & 10,50 \\
\hline & $\begin{array}{l}\hat{y}=0,769+0,025^{(0,232)} \mathrm{a} \\
\hat{y}=\mathrm{b}_{2}=0,779\end{array}$ & $\begin{array}{l}\forall 0,091 \leq \mathrm{a} \leq 0,417 \mathrm{~m}^{2} \\
\forall 0,417 \leq \mathrm{a} \leq 11,052 \mathrm{~m}^{2}\end{array}$ & 0,993 & 0,417 \\
\hline & $\begin{array}{l}\hat{s}=0,023+0,024^{* *} \mathrm{a} \\
\hat{s}=\mathrm{b}_{2}=0,041\end{array}$ & $\begin{array}{l}\forall 0,091 \leq \mathrm{a} \leq 0,722 \mathrm{~m}^{2} \\
\forall 0,722 \leq \mathrm{a} \leq 11,052 \mathrm{~m}^{2}\end{array}$ & 0,957 & 0,722 \\
\hline
\end{tabular}

Para P no PCDA, a dimensão do indivíduo foi de $1,209 \mathrm{~m}^{2}$ x $0,1 \mathrm{~m}$, baseada no início do platô para a fertilidade média (Quadro 4). A ausência de pontos de concentração do nutriente a curtas distâncias, no momento de coleta das amostras simples, fez com que havesse maior uniformidade do $\mathrm{P}$ no solo após a aração, iniciando-se o platô em dimensões maiores do que para o PD e para o PCAA.

Para os teores de potássio (K), não foi possível selecionar um modelo no PD (Quadro 4). A permanência de muitas linhas dos cultivos anteriores na área, além de lavagem do K a partir da parte área das plantas de milho, como sugerido por Klepker \& Anghinoni (1995), pode explicar uma distribuição mais uniforme de K, impedindo a formação de um platô. Porém, para o PCAA e para o PCDA, o platô foi atingido com 0,540 e $1,283 \mathrm{~m}^{2} \times 0,1 \mathrm{~m}$, respectivamente, considerando os desvios-padrão dos teores desta característica (Quadro 4). Para as médias, as dimensões das unidades de amostra foram de 1,856 e de $1,712 \mathrm{~m}^{2}$ x $0,1 \mathrm{~m}$, no PCAA e no PCDA, respectivamente (Quadro 4). Como já explicado, deve ficar claro que a dimensão que melhor represente a variabilidade é mais importante para a definição da dimensão "ideal" da unidade de amostra do que a que represente o teor médio local de determinada característica.

Para os teores de $\mathrm{Ca}^{2+}$ e de $\mathrm{Mg}^{2+}$, ambos os nutrientes aplicados na forma de corretivo, uniformemente distribuído, a dimensão do indivíduo, em alguns casos, foi excessivamente elevada ou a variabilidade apresentou aumento linear sem estabilização, não configurando uma variação a curtas distâncias no solo (0,05 a 2 m), especialmente no PD (Quadro 4). A maior semelhança entre a distribuição das duas características avaliadas $\left(\mathrm{Ca}^{2+} \mathrm{e} \mathrm{Mg}^{2+}\right)$ se deu no PCDA, provavelmente por efeito da aração, o que proporcionou dimensões para as unidades de amostra de solo muito próximas (Quadro 4).

As dimensões definidas com base nos desviospadrão de $\mathrm{H}+\mathrm{Al}$ foram pequenas, exceto no $\mathrm{PD}$ onde a variabilidade dos valores de $\mathrm{H}+\mathrm{Al}$ apresentou aumento linear sem formação de platô (Quadro 5).

Com exceção de fósforo remanescente (P-rem) no PCAA, as dimensões dos indivíduos selecionadas para matéria orgânica e P-rem não se enquadram na definição de indivíduo solo apresentada no presente trabalho, pois a dimensão relativa à formação do platô, quando este existiu, não configurou uma variação a curtas distâncias no solo (0,05 a 2 m) (Quadro 5).

$\mathrm{O}$ indivíduo solo, dimensionado com base nos desvios-padrão dos valores de $\mathrm{pH}$, foi praticamente o dobro no PCAA em relação PCDA (Quadro 5), uma vez que a aração recente à coleta de amostras pode ter provocado maior variabilidade dos valores de $\mathrm{pH}$ a curtas distâncias no solo no PCDA. Santos \& Vasconcellos (1987) mostraram que a variabilidade do $\mathrm{pH}$ no solo pode aumentar após a aração, sendo sua distribuição mais homogênea antes do preparo.

Apesar da grande variação na dimensão das unidades de amostra, conforme a característica da fertilidade do solo avaliada e o tipo de situação de manejo-coleta, podem-se desconsiderar as dimensões das unidades de amostra que não configuraram uma variação a curtas distâncias no solo (semidiagonal > $106,25 \mathrm{~cm}$, ou seja, dimensão $>2,933 \mathrm{~m}^{2}$ x 0,1 m) e aquelas estimadas para as médias das características avaliadas. Dessa forma, a dimensão do indivíduo solo pode ser resumida à apresentada pelos desvios-padrão 
Quadro 5. Equações de regressão das médias ${ }^{(1)}$ dos valores de H + Al, matéria orgânica, P-rem e pH e das médias ${ }^{(1)}$ dos desvios-padrão destes valores $\left(\mathrm{cmol}_{\mathrm{c}} \mathrm{dm}^{-3}, \mathrm{dag} \mathrm{kg}^{-1} \mathrm{e} \mathrm{mg} \mathrm{L}^{-1}\right)$, considerando a área da unidade de amostra de solo e área dimensionada para a unidade de amostra em forma de prisma hexagonal, para uma profundidade de $10 \mathrm{~cm}$, em três situações de manejo-coleta

\begin{tabular}{|c|c|c|c|c|}
\hline Situação(2) & \multicolumn{2}{|c|}{ Equações de regressão } & $\mathbf{R}^{2}$ & $\operatorname{Area}\left(\mathrm{m}^{2}\right)$ \\
\hline \multicolumn{5}{|c|}{$\mathrm{H}+\mathrm{Al}$} \\
\hline & $\begin{array}{l}\hat{y}=3,087-0,080^{(0,257)} \mathrm{a} \\
\hat{y}=\mathrm{b}_{2}=3,014\end{array}$ & $\begin{array}{l}\forall 0,091 \leq \mathrm{a} \leq 0,918 \mathrm{~m}^{2} \\
\forall 0,918 \leq \mathrm{a} \leq 11,052 \mathrm{~m}^{2}\end{array}$ & 0,831 & 0,918 \\
\hline & $\hat{s}=0,392+0,014^{* *} \mathrm{a}$ & $\forall 0,091 \leq \mathrm{a} \leq 11,052 \mathrm{~m}^{2}$ & 0,982 & 11,052 \\
\hline \multirow[t]{2}{*}{ PCAA } & $\begin{array}{l}\hat{y}=6,405+0,308^{* 2} \mathrm{a} \\
\hat{y}=\mathrm{b}_{2}=6,717\end{array}$ & $\begin{array}{l}\forall 0,091 \leq \mathrm{a} \leq 1,012 \mathrm{~m}^{2} \\
\forall 1,012 \leq \mathrm{a} \leq 11,052 \mathrm{~m}^{2}\end{array}$ & 0,925 & 1,012 \\
\hline & $\begin{array}{l}\hat{s}=0,387+0,473^{*} \mathrm{a} \\
\hat{s}=\mathrm{b}_{2}=0,687\end{array}$ & $\begin{array}{l}\forall 0,091 \leq \mathrm{a} \leq 0,634 \mathrm{~m}^{2} \\
\forall 0,634 \leq \mathrm{a} \leq 11,052 \mathrm{~m}^{2}\end{array}$ & 0,917 & 0,634 \\
\hline \multirow[t]{2}{*}{ PCDA } & $\begin{array}{l}\hat{y}=3,769+0,145^{* *} \mathrm{a} \\
\hat{y}=\mathrm{b}_{2}=3,914\end{array}$ & $\begin{array}{l}\forall 0,091 \leq \mathrm{a} \leq 1,003 \mathrm{~m}^{2} \\
\forall 1,003 \leq \mathrm{a} \leq 11,052 \mathrm{~m}^{2}\end{array}$ & 0,996 & 1,003 \\
\hline & $\begin{array}{l}\hat{S}=0,241+0,052^{(0,293)} \mathrm{a}-0,021^{(0,400)} \mathrm{a}^{2} \\
\hat{S}=\mathrm{b}_{2}=0,265\end{array}$ & $\begin{array}{l}\forall 0,091 \leq \mathrm{a} \leq 0,722 \mathrm{~m}^{2} \\
\forall 0,722 \leq \mathrm{a} \leq 11,052 \mathrm{~m}^{2}\end{array}$ & 0,983 & 0,615 \\
\hline \multicolumn{5}{|c|}{ Matéria orgânica } \\
\hline \multirow[t]{2}{*}{$\mathrm{PD}$} & $\begin{array}{l}\hat{y}=4,321-0,090^{(0,146)} \mathrm{a}+0,029^{(0,141)} \mathrm{a}^{2} \\
\hat{y}=\mathrm{b}_{2}=4,334\end{array}$ & $\begin{array}{l}\forall 0,091 \leq \mathrm{a} \leq 3,211 \mathrm{~m}^{2} \\
\forall 3,211 \leq \mathrm{a} \leq 11,052 \mathrm{~m}^{2}\end{array}$ & 0,986 & 3,211 \\
\hline & $\begin{array}{l}\hat{s}=0,663-0,080^{\circ} \mathrm{a}+0,019^{\circ} \mathrm{a}^{2} \\
\hat{s}=\mathrm{b}_{2}=0,635\end{array}$ & $\begin{array}{l}\forall 0,091 \leq \mathrm{a} \leq 3,814 \mathrm{~m}^{2} \\
\forall 3,814 \leq \mathrm{a} \leq 11,052 \mathrm{~m}^{2}\end{array}$ & 0,990 & 3,814 \\
\hline \multirow[t]{2}{*}{ PCAA } & $\hat{y}=\bar{y}=3,687$ & $\forall 0,091 \leq \mathrm{a} \leq 11,052 \mathrm{~m}^{2}$ & & 0,091 \\
\hline & $\hat{s}=\bar{y}=0,502$ & $\forall 0,091 \leq \mathrm{a} \leq 11,052 \mathrm{~m}^{2}$ & & 0,091 \\
\hline \multirow[t]{2}{*}{ PCDA } & $\hat{y}=\bar{y}=2,773$ & $\forall 0,091 \leq \mathrm{a} \leq 11,052 \mathrm{~m}^{2}$ & & 0,091 \\
\hline & $\begin{array}{l}\hat{s}=0,538-0,009^{(0,227)} \mathrm{a} \\
\hat{s}=\mathrm{b}_{2}=0,494\end{array}$ & $\begin{array}{l}\forall 0,091 \leq \mathrm{a} \leq 4,956 \mathrm{~m}^{2} \\
\forall 4,956 \leq \mathrm{a} \leq 11,052 \mathrm{~m}^{2}\end{array}$ & 0,837 & 4,956 \\
\hline \multicolumn{5}{|c|}{ P-rem } \\
\hline \multirow[t]{2}{*}{ PD } & $\hat{y}=\bar{y}=27,936$ & $\forall 0,091 \leq \mathrm{a} \leq 11,052 \mathrm{~m}^{2}$ & & 0,091 \\
\hline & $\begin{array}{l}\hat{s}=1,571+0,100^{\star \star} \mathrm{a} \\
\hat{s}=\mathrm{b}_{2}=1,971\end{array}$ & $\begin{array}{l}\forall 0,091 \leq \mathrm{a} \leq 4,009 \mathrm{~m}^{2} \\
\forall 4,009 \leq \mathrm{a} \leq 11,052 \mathrm{~m}^{2}\end{array}$ & 0,955 & 4,009 \\
\hline \multirow[t]{2}{*}{ PCAA } & $\hat{y}=\bar{y}=23,964$ & $\forall 0,091 \leq \mathrm{a} \leq 11,052 \mathrm{~m}^{2}$ & & 0,091 \\
\hline & $\begin{array}{l}\hat{s}=1,238+0,312^{\star} \mathrm{a} \\
\hat{s}=\mathrm{b}_{2}=1,525\end{array}$ & $\begin{array}{l}\forall 0,091 \leq \mathrm{a} \leq 0,920 \mathrm{~m}^{2} \\
\forall 0,920 \leq \mathrm{a} \leq 11,052 \mathrm{~m}^{2}\end{array}$ & 0,916 & 0,920 \\
\hline \multirow[t]{2}{*}{ PCDA } & $\begin{array}{l}\hat{y}=22,260+0,361^{\star \star} \mathrm{a} \\
\hat{y}=\mathrm{b}_{2}=22,620\end{array}$ & $\begin{array}{l}\forall 0,091 \leq \mathrm{a} \leq 0,996 \mathrm{~m}^{2} \\
\forall 0,996 \leq \mathrm{a} \leq 11,052 \mathrm{~m}^{2}\end{array}$ & 0,971 & 0,996 \\
\hline & $\begin{array}{l}\hat{S}=0,910+0,031^{\text {** }} \mathrm{a} \\
\hat{s}=\mathrm{b}_{2}=1,062\end{array}$ & $\begin{array}{l}\forall 0,091 \leq \mathrm{a} \leq 0,722 \mathrm{~m}^{2} \\
\forall 0,722 \leq \mathrm{a} \leq 11,052 \mathrm{~m}^{2}\end{array}$ & 0,902 & 4,878 \\
\hline
\end{tabular}

de P no PCAA e de K no PCDA (Quadro 4). Estas foram as maiores dimensões encontradas, considerando o conceito de indivíduo solo apresentado; pode-se admitir, então, que uma unidade de amostra com a dimensão de, aproximadamente, 1,283 $\mathrm{m}^{2} \times 0,1 \mathrm{~m}$ possa incluir as variações a curtas distâncias no solo e estimar adequadamente a fertilidade local média para todas as situações de manejo-coleta estudadas (PD, PCAA e PCDA). No entanto, para que haja concordância com os modelos criados para o indivíduo solo (Figura 2), a dimensão da unidade de amostra deve apresentar exatamente $1,228 \mathrm{~m}^{2}$ x $0,1 \mathrm{~m}$.

Posteriormente, foi calculado o número de amostras simples necessário para a caracterização da unidade de amostra de solo com esta dimensão, utilizando-se os resultados obtidos no início dos platôs das equações selecionadas para P disponível no PD e no PCAA, uma vez que esta característica apresentou maior 
Quadro 5. Continuação

\begin{tabular}{|c|c|c|c|c|}
\hline Situação(2) & \multicolumn{2}{|c|}{ Equações de regressão } & $\mathbf{R}^{2}$ & Area $\left(m^{2}\right)$ \\
\hline \multicolumn{5}{|c|}{$\mathrm{pH}$} \\
\hline \multirow[t]{2}{*}{ PD } & $\begin{array}{l}\hat{y}=6,309+0,083^{\star} \mathrm{a} \\
\hat{y}=\mathrm{b}_{2}=6,385\end{array}$ & $\begin{array}{l}\forall 0,091 \leq \mathrm{a} \leq 0,919 \mathrm{~m}^{2} \\
\forall 0,919 \leq \mathrm{a} \leq 11,052 \mathrm{~m}^{2}\end{array}$ & 0,964 & 0,919 \\
\hline & $\hat{s}=0,5380-0,009^{2 z} \mathrm{a}$ & $\forall 0,091 \leq \mathrm{a} \leq 11,052 \mathrm{~m}^{2}$ & 0,952 & 11,052 \\
\hline \multirow[t]{2}{*}{ PCAA } & $\hat{y}=\bar{y}=5,007$ & $\forall 0,091 \leq \mathrm{a} \leq 11,052 \mathrm{~m}^{2}$ & & 0,091 \\
\hline & $\begin{array}{l}\hat{s}=0,166+0,110^{*} \mathrm{a} \\
\hat{s}=\mathrm{b}_{2}=0,285\end{array}$ & $\begin{array}{l}\forall 0,091 \leq \mathrm{a} \leq 1,080 \mathrm{~m}^{2} \\
\forall 1,080 \leq \mathrm{a} \leq 11,052 \mathrm{~m}^{2}\end{array}$ & 0,952 & 1,080 \\
\hline \multirow[t]{2}{*}{ PCDA } & $\begin{array}{l}\hat{y}=5,596+0,148^{\circ} \mathrm{a}-0,218^{\circ} \mathrm{a}^{2} \\
\hat{y}=\mathrm{b}_{2}=5,617\end{array}$ & $\begin{array}{l}\forall 0,091 \leq \mathrm{a} \leq 0,191 \mathrm{~m}^{2} \\
\forall 0,191 \leq \mathrm{a} \leq 11,052 \mathrm{~m}^{2}\end{array}$ & 0,959 & 0,191 \\
\hline & $\begin{array}{l}\hat{s}=0,077-0,121^{(0,296)} \mathrm{a}+0,203^{(0,283)} \mathrm{a}^{2} \\
\hat{s}=\mathrm{b}_{2}=0,072\end{array}$ & $\begin{array}{l}\forall 0,091 \leq \mathrm{a} \leq 4,956 \mathrm{~m}^{2} \\
\forall 4,956 \leq \mathrm{a} \leq 11,052 \mathrm{~m}^{2}\end{array}$ & 0,971 & 0,553 \\
\hline
\end{tabular}

\footnotetext{
${ }^{(1)}$ Médias de cinco indivíduos-solo (cinco hexágonos de amostragem) $\left(\bar{y}_{\mathrm{ij}}\right.$ e $\left.{ }_{\mathrm{ij}}\right){ }^{(2)} \mathrm{PD}=$ plantio direto, PCAA = plantio convencional antes da aração e PCDA = plantio convencional depois da aração. ${ }^{()}$, * $\mathrm{e}^{* *}$, significativo ao valor indicado entre parênteses, a 5 e a $1 \%$, respetivamente.
}

variabilidade dentre todas as estudadas. Para fins de comparação, foi também calculado o número de amostras simples necessário para representar o talhão homogêneo onde se realizou a amostragem no PD e no PCAA, utilizando-se a variabilidade de $\mathrm{P}$ disponível estimada pelo conjunto de 275 amostras simples coletadas em cada situação (Quadro 6).

O número de amostras simples necessário para representar o indivíduo solo foi elevado e muito semelhante ao número necessário para formar uma amostra composta representativa do talhão homogêneo onde se realizou a amostragem, nas duas situações de manejo-coleta (PD e PCAA) (Quadro 6). A partir deste resultado, pode-se inferir que a estimativa da variabilidade interna do indivíduo, com dimensão de $1,228 \mathrm{~m}^{2} \times 0,1 \mathrm{~m}$, pode apresentar magnitude semelhante à variabilidade estimada para toda a população, demonstrando que a variabilidade é intensa e ocorre a curtas distâncias no solo, como observado por van den Hende \& Cottenie (1960); Jackson (1976); Alvarez V. \& Carraro (1976); Saraiva et al. (1992); Raun et al. (1998).

Para Jackson (1976), a variabilidade a curtas distâncias no solo não dificulta o crescimento e o desenvolvimento das plantas, uma vez que a extensão do sistema radicular é suficiente para explorar áreas com diferentes teores de nutrientes, justificando a avaliação da fertilidade média do solo. Portanto, na prática, a obtenção de uma amostra composta por indivíduo solo, para a avaliação da fertilidade média do solo, pode ser questionável, em virtude do custos, pois, com praticamente o mesmo número de amostras simples (Quadro 6), pode-se formar uma amostra composta que caracterize, adequadamente, a fertilidade média de todo o talhão homogêneo (unidade de amostragem).
Quadro 6. Número de amostras simples necessário para a caracterização das unidades de amostra de solo com dimensão de $1,228 \mathrm{~m}^{2} \times 0,1 \mathrm{~m}$ e número de amostras simples necessário para a representação da unidade de amostragem (talhão homogêneo), utilizando os resultados de $P$ disponível, em duas situações de manejocoleta, para $f=10$ e $20 \%$

\begin{tabular}{|c|c|c|c|c|}
\hline \multirow{3}{*}{ Tolerância } & \multicolumn{4}{|c|}{ Situação(1) } \\
\hline & \multicolumn{2}{|l|}{ PD } & \multicolumn{2}{|c|}{ PCAA } \\
\hline & Indivíduo & Talhão & Indivíduo & Talhão \\
\hline & & $r$ & (2) & + \\
\hline $\begin{array}{l}f=10 \% \\
f=20 \%\end{array}$ & $\begin{array}{r}154 \\
39\end{array}$ & 198 & 80 & 82 \\
\hline $\mathrm{f}=20 \%$ & 39 & 49 & 20 & 20 \\
\hline
\end{tabular}

(1) $\mathrm{PD}=$ plantio direto; $\mathrm{PCAA}=$ plantio convencional antes da aração. ${ }^{(2)} \mathrm{n}=\left(\left(\mathrm{t} \alpha_{/ 2} \mathrm{CV}\right) / \mathrm{f}\right)^{2}, \alpha=0,05 \mathrm{e}\left(\mathrm{n}_{\mathrm{i}}-1\right)$, sendo: $\mathrm{n}_{\mathrm{i}}-1=24$ para o indivíduo (25 amostras simples coletadas na dimensão mais próxima da selecionada) e $\cong \infty$ para o talhão (275 amostras simples por situação de manejo-coleta).

A agricultura de precisão comercial, no entanto, operando em áreas de 10 ou até 1.000 ha, tem utilizado grids de amostragem com distâncias entre amostras simples de $100 \mathrm{~m}$ ou mais, com pequena evidência científica de adequabilidade (Sadler et al., 1998). Além disso, por causa dos custos, são coletadas apenas algumas amostras simples (seis a oito, geralmente com pequeno volume), sendo analisadas individualmente (Anderson-Cook et al., 1999). Desta forma, pode-se 
inferir que os mapas de fertilidade gerados podem não contemplar a real variabilidade da fertilidade do solo, pois cada ponto de amostragem irá representar as concentrações localizadas de nutrientes, e estas podem ser extremamente variáveis em distâncias menores que $1 \mathrm{~m}$, como demonstrado pelos resultados apresentados neste estudo.

Para contemplar as variações a curtas distâncias no solo, uma vez que na agricultura de precisão não se procura a fertilidade média, os grids de amostragem deveriam ser menores, tornando-se, porém, antieconômicos (se a distância entre pontos fosse de $1 \mathrm{~m}$, seriam analisadas individualmente 10.000 amostras em 1 ha) (Raun et al., 1998).

A questão econômica, entretanto, não é o único problema neste caso. A redução no espaçamento dos grids de amostragem pode gerar padrões cíclicos (tendências) de diferentes magnitudes, os quais, muitas vezes, afetam marcadamente a forma do semivariograma (modelo simples para o semivariograma pode não ser adequado), além de aumentar a ocorrência de "outliers", especialmente no plantio direto (Mallarino, 1996). Para Mulla \& McBratney (2000), os "outliers" podem afetar significativamente a forma do semivariograma, diminuindo o alcance mesmo na presença de elevada dependência espacial.

As variações a curtas distâncias podem ser atenuadas com o uso do "bulking" (retirada de muitas amostras simples de pequeno volume e obtenção de médias locais, ou formação de amostras compostas a partir destas "amostras simples") (Webster \& Burgess, 1984; Burrough, 1991; Mulla \& McBratney, 2000). $\mathrm{O}$ indivíduo solo (unidade de amostra), caracterizado no presente estudo, pode ser utilizado como "bulking", apresentando a vantagem de ter sido dimensionado para representar a fertilidade efetivamente explorada por uma planta ou por um grupo de plantas (fertilidade local média), além de apresentar dimensões laterais estritamente necessárias para incluir variações representativas a curtas distâncias na composição do solo (mesovariações).

Para Cahn et al. (1994), os esquemas de amostragem precisam ser flexíveis, para caracterizar, acuradamente, a dependência espacial de várias características do solo e, provavelmente, o esquema de amostragem mais flexível seja a coleta de amostras compostas em áreas de $1 \mathrm{~m}^{2}$, distribuídas ao longo do campo de cultivo. A área sugerida pelo autor é muito próxima da área selecionada para o indivíduo solo $\left(1,228 \mathrm{~m}^{2}\right)$, o que reforça, mais uma vez, sua aplicabilidade prática na agricultura de precisão.

Para confirmar, foram calculados os coeficientes de variação (CV) entre os valores das características determinadas nas amostras simples centrais de cada hexágono de amostragem (cinco hexágonos) e entre as médias locais obtidas com as unidades de amostra de solo. Preferiu-se, para tanto, um indivíduo "real", utilizado na amostragem de solo no estudo $\left(0,822 \mathrm{~m}^{2} \times 0,1 \mathrm{~m}\right)$, porém, com a dimensão mais próxima possível da unidade de amostra selecionada (1,228 $\mathrm{m}^{2}$ x 0,1 m) (Quadro 7).

A maioria das características avaliadas apresentou redução na variabilidade, quando se utilizaram as médias locais das unidades de amostra de solo, em relação aos valores determinados nas amostras simples centrais de cada hexágono de amostragem, sendo este fato mais marcante para $\mathrm{P}$ disponível e $\mathrm{P}$ rem no $\mathrm{PD}$, para $\mathrm{K}$ no $\mathrm{PD}$ e no $\mathrm{PCDA}$ e para matéria orgânica nas três situações de manejo-coleta (Quadro 7). A redução na variabilidade significa menor influência das variações a curtas distâncias, o que pode atenuar a ocorrência de padrões cíclicos e de "outliers", melhorando a forma do semivariograma e aumentando o alcance. Além disso, a confiabilidade

Quadro 7. Coeficientes de variação de características químicas da fertilidade determinadas nas amostras simples centrais (a.s.) de cada um dos cinco hexágonos de amostragem e de médias locais ${ }^{(1)}$ obtidas nas unidades de amostra (u.a.) de $\left(0,822 \mathrm{~m}^{2} \times 0,1 \mathrm{~m}\right)$, em três situações de manejo-coleta

\begin{tabular}{|c|c|c|c|c|c|c|}
\hline \multirow{2}{*}{ Característica } & \multicolumn{2}{|c|}{$\mathbf{P D}^{(2)}$} & \multicolumn{2}{|c|}{ PCAA } & \multicolumn{2}{|c|}{ PCDA } \\
\hline & a.s. & u.a. & a.s. & u.a. & a.s. & u.a. \\
\hline $\mathrm{P}$ & 64,19 & 36,33 & 26,17 & 23,36 & 10,32 & 7,96 \\
\hline $\mathrm{K}$ & 26,91 & 9,09 & 12,53 & 7,83 & 30,60 & 10,29 \\
\hline $\mathrm{Ca}^{2+}$ & 16,75 & 15,11 & 7,78 & 4,08 & 11,70 & 6,13 \\
\hline $\mathrm{Mg}^{2+}$ & 6,86 & 8,73 & 16,56 & 12,96 & 11,25 & 9,24 \\
\hline $\mathrm{H}+\mathrm{Al}$ & 27,70 & 30,43 & 8,50 & 7,89 & 11,57 & 9,11 \\
\hline MO & 18,30 & 9,97 & 16,34 & 7,95 & 34,68 & 11,05 \\
\hline P-rem & 7,50 & 3,15 & 15,52 & 11,08 & 9,25 & 6,75 \\
\hline $\mathrm{pH}$ & 6,05 & 6,15 & 3,49 & 2,99 & 1,92 & 1,25 \\
\hline
\end{tabular}

(1) Média de cada hexágono de amostragem. ${ }^{(2)} \mathrm{PD}=$ Plantio direto; PCAA = Plantio convencional antes da aração; PCDA = Plantio convencional depois da aração. 
dos mapas de fertilidade será aumentada, por estar sendo considerada a fertilidade efetivamente explorada por uma planta ou por um grupo de plantas (fertilidade local média). Estes benefícios podem ser conseguidos sem aumentar o número de amostras a serem analisadas, bastando para isso, analisar a amostra composta representativa do indivíduo solo (unidade de amostra).

Dessa forma, o único problema seria o elevado número de amostras simples necessário para representar a unidade de amostra de solo selecionada $\left(1,228 \mathrm{~m}^{2} \times 0,1 \mathrm{~m}\right)$, especialmente para $\mathrm{P}$ no PD (Quadro 6), o que pode aumentar o trabalho e, ou, o custo, necessários para realizar a amostragem do solo. Entretanto, o indivíduo de $\left(0,822 \mathrm{~m}^{2} \times 0,1 \mathrm{~m}\right)$, composto originalmente por 25 amostras simples, gerou uma redução na variabilidade de $\mathrm{P}$ e $\mathrm{K}$ no $\mathrm{PD}$ de 43 e $66 \%$, respectivamente, e de 38 e $66 \%$ para K no PCAA e no PCDA, respectivamente, em relação às amostras simples coletadas no centro dos hexágonos (Quadro 7). Dessa forma, acredita-se que este número de amostras simples $(\mathrm{n}=25)$ substitua o número inicialmente calculado $(\mathrm{n}=39)$ (Quadro 6), devendose aceitar, para tanto, um desvio em torno da média (f) de 24,8 e $17,8 \%$, para $\mathrm{P}$ no PD e no PCAA, respectivamente.

Assim, a unidade de amostra de solo que poderia satisfazer o maior número de situações com aplicabilidade prática para a agricultura de precisão seria caracterizada por 25 amostras simples coletadas num hexágono de $68,75 \mathrm{~cm}$ de lado e área de 1,228 $\mathrm{m}^{2}$, distribuídas aleatoriamente ou de forma sistemática, como a utilizada no presente estudo.

\section{CONCLUSÕES}

1. A estimativa da variabilidade interna do indivíduo solo selecionado, com dimensão de $1,228 \mathrm{~m}^{2} \times 0,1 \mathrm{~m}$, apresentou magnitude semelhante à variabilidade estimada para toda a população (unidade de amostragem), demonstrando que a variabilidade é intensa e ocorre a curtas distâncias no solo.

2. Para a caracterização do indivíduo solo (unidade de amostra), devem-se coletar 25 amostras simples, necessárias à formação de uma amostra composta representativa, num hexágono de $68,75 \mathrm{~cm}$ de lado e área de $1,228 \mathrm{~m}^{2}$.

\section{LITERATURA CITADA}

ALVAREZ V., V.H. \& ALVAREZ, G.A.M. Apresentação de equações de regressão e suas interpretações. B. Inf. SBCS, 28:28-32,2003.

ALVAREZ V., V.H. \& CARRARO, I.M. Variabilidade do solo numa unidade de amostragem em solos de Cascavel e de Ponta Grossa, Paraná. R. Ceres, 23:503-510, 1976.
ALVAREZ V., V.H.; NOVAIS, R.F.; DIAS, L.E. \& OLIVEIRA, J.A. Determinação e uso do fósforo remanescente, B. Inf. SBCS, 52:27-32, 2000.

ANDERSON-COOK, C.M.; ALLEY, M.M.; NOBLE, R. \& KHOSLA, R. Phosphorus and potassium fertilizer recommendation variability for two mid-atlantic coastal plain fields. Soil Sci. Soc. Am. J., 63:1740-1747, 1999.

BURROUGH, P.A. Sampling designs for quantifying map unit composition. In: MUSBACH, M.J. \& WILDING, L.P., eds. Spatial variabilities of soil and landforms. Soil Sci. Soc. Am., 1991. p.89-125. (SSSA Special Publication, 28).

CAHN, M.D.; HUMMEL, J.W. \& BROUER, B.H. Spatial analysis of soil fertility for site-specific crop management. Soil Sci. Soc. Am. J., 58:1240-1248, 1994.

COCHRAN, W.G. Técnicas de amostragem. Rio de Janeiro, Fundo de Cultura, 1965. 555p.

DEFELIPO, B.V. \& RIBEIRO, A.C. Análise química do solo (metodologia). Viçosa, MG, Universidade Federal Viçosa, 1996. 17p. (Boletim de Extensão, 29)

JACKSON, M.L. Analisis quimico de suelos. Barcelona, Omega, 1976. 662p.

KLEPKER, D. \& ANGHINONI, I. Características físicas e químicas do solo afetadas por métodos de preparo e modos de adubação. R. Bras. Ci. Solo, 19:395-401, 1995.

MALLARINO, A.P. Spatial variability patterns of phosphorus and potassium in no-tilled for two sampling scales. Soil Sci. Soc. Am. J., 60:1473-1481, 1996.

MULLA, D.J. \& McBRATNEY, A.B. Soil spatial variability. In: SUMNER, M.E., ed. Handbook of Soil Science. New York, CRC Press, 2000. p.321-352.

RAUN, W.R.; SOLIE, J.B.; JOHNSON,G.V.; STONE, M.L.; WHITNEY, R.W.; LEES, H.L.; SEMBIRING, H. \& PHILLIPS, S.B. Microvariability in soil test, plant nutrient, and yield parameters in bermudagrass. Soil Sci. Soc. Am. J., 62:683-690, 1998.

SADLER, J.E.; BUSSCHER, W.J.; BAVER, P.J. \& KARLEN, D.L. Spatial scale requirements for precision farming: A case study in the South Eastern USA. Agron. J. 90:191197, 1998.

SANTOS, H.L \& VASCONCELOS, C.A. Determinação do número de amostras de solo para análise química em diferentes condições de manejo. R.Bras. Ci. Solo, 11:97100, 1987.

SARAIVA, O.F.; ALVAREZ V., V.H. \& COSTA, L.M. Variabilidade de algumas características físicas e químicas de um Podzólico Vermelho-Amarelo Câmbico distrófico. R. Ceres, 39:529-541, 1992.

van den HENDE, A. \& COTTENIE, A. L'estimation de la fertilité du sol par les méthodes chimiques nouvelles. In: Comptes Rendus Des Recherches.; Travaux du Centre de Chemie Physique Agricole. Bruxelles, IRSIA, 1960. p.27-174. (Bulletin, 25)

WEBSTER, R. \& BURGESS, T.M. Sampling and bulking strategies for estimating soil properties in small regions. J. Soil Sci., 35:127-140, 1984. 